\title{
红外光谱技术在淀粉粒有序结构分析中的应用
}

\author{
满建民 $^{1} \quad$ 蔡灿辉 $^{1}$ 严秋香 $^{2}$ 胡茂志 ${ }^{2}$ 刘巧泉 ${ }^{1, *}$ 韦存虚 $^{1, *}$ \\ ${ }^{1}$ 扬州大学教育部植物功能基因组学重点实验室 / 江苏省作物遗传生理重点实验室, 江苏扬州 $225009 ;{ }^{2}$ 扬州大学测试中心, 江苏 \\ 扬州 225009
}

摘 要: 傅里叶变换红外光谱技术(FTIR)可用于研究淀粉粒的有序结构, 包括透射模式和衰减全反射模式 2 种。本文 探讨不同去卷积设置条件对 FTIR 波谱的影响, 并分析 FTIR 在淀粉粒有序结构分析中的应用。研究结果表明, 不同 去卷积设置对 FTIR 波谱和相关峰强度影响较大, 以半峰宽 $19 \mathrm{~cm}^{-1}$ 和增强因子 1.9 的设置对 FTIR 原始波谱去卷积, 获得的结果较好。天然淀粉晶体类型不同, 其 FTIR 波谱有差异, 表现在马铃薯和山药淀粉的衰减全反射 FTIR 波谱 相似, 与水稻淀粉明显不同; 水稻和马铃薯淀粉透射 FTIR 波谱相似，与山药淀粉明显不同。淀粉中的水分含量影响 衰减全反射 FTIR 波谱, 当水分含量超过 $60 \%$ 时, 对波谱分析结果基本没有影响。酸水解优先降解淀粉粒无定形区的 结构成分, 提高淀粉粒的有序度。淀粉葡糖苷酶水解淀粉对淀粉粒外部区域的有序度影响不大, 但明显提高整个淀粉 粒的有序度。不同品质稻米淀粉的衰减全反射 FTIR 波谱相似。上述研究结果为应用 FTIR 分析淀粉粒有序结构提供 重要的参考作用。

关键词: 傅里叶变换红外光谱; 淀粉粒; 有序结构; 波谱去卷积

\section{Applications of Infrared Spectroscopy in the Analysis of Ordered Structure of Starch Grain}

\author{
MAN Jian-Min ${ }^{1}$, CAI Can-Hui ${ }^{1}$, YAN Qiu-Xiang ${ }^{2}$, HU Mao-Zhi ${ }^{2}$, LIU Qiao-Quan ${ }^{1, *}$, and WEI Cun-Xu ${ }^{1, *}$ \\ ${ }^{1}$ Key Laboratories of Plant Functional Genomics of the Ministry of Education and Crop Genetics and Physiology of the Jiangsu Province, Yangzhou \\ University, Yangzhou 225009, China; ${ }^{2}$ Testing Center, Yangzhou University, Yangzhou 225009, China
}

\begin{abstract}
Fourier transform infrared spectroscopy (FTIR) is used to study the ordered structure of starch grain, which has two modes: transmittance and attenuated total reflectance (ATR). In this paper, the different deconvolution parameters of spectra were applied to compare their effects on FTIR spectra in studying the ordered structure of starch grain. The results indicated that the different deconvolution parameters had significant effects on FTIR spectra and the intensities of relative peaks. The peak half-width of $19 \mathrm{~cm}^{-1}$ and the resolution enhancement factor of 1.9 were ideal deconvolution parameters of spectra to obtain the better results. Native starches had A, B, and C three types of crystalline, their FTIR spectra showed some differences. Potato and Chinese yam starches had similar ATR-FTIR spectra, which were different from that of rice starch. However, rice and potato starches had similar transmittance-FTIR spectra, which were different from that of Chinese yam starch. The water content of sample affected the spectra of ATR-FTIR, but this effect was not detected when water content exceeded 60\%. The ATR-FTIR spectra showed that the hydrolysis of amorphous structure in starch grain was faster than that of ordered structure during acid treatment. The ordered degree of structure in starch grain increased with increasing time of acid hydrolysis. The amyloglucosidase hydrolysis had no significant effect on the ordered degree of structure at the outside of starch grain by the ATR-FTIR spectra, but the ordered degree of structure of whole starch grain significantly increased with enzyme hydrolysis according to the transmittance-FTIR spectra. The amylose content is an important physicochemical property in determining the starch quality. Rice starches with different amylose contents showed the similar ATR-FTIR spectra. These results would be very useful for the application of FTIR to the analysis of ordered structure of starch grain.
\end{abstract}

Keywords: Fourier transform infrared spectroscopy; Starch grain; Ordered structure; Deconvolution of spectrum

本研究由国家自然科学基金项目(31071342)和江苏省作物学优势学科项目资助。

* 通讯作者(Corresponding authors): 刘巧泉, E-mail: qqliu@yzu.edu.cn; 韦存虚, E-mail: cxwei@yzu.edu.cn Received(收稿日期): 2011-08-22; Accepted(接受日期): 2011-12-15; Published online(网络出版日期): 2012-01-04. URL: http://www.cnki.net/kcms/detail/11.1809.S.20120104.1649.009.html 
淀粉是植物主要的能量䛎藏物质,也是重要的 食品来源和工业原料。植物淀粉以半晶态的颗粒形 式存在于自然界, 包含结晶区和无定形区 2 种结构 成分, 主要由直链淀粉和支链淀粉组成 ${ }^{[1]}$ 。淀粉分子 中的直链淀粉和支链淀粉中的短链部分形成了双螺 旋结构, 又称为短程有序结构 (short-range ordered structure), 这些双螺旋分子链通过分子间的相互作 用力以一定的空间点阵在淀粉颗粒的某些区域形成 不同的多晶形, 即晶体, 又称为长程有序结构(longrange ordered structure $)^{[1-2]}$ 。依据粉末 X-射线衍射波 谱, 可将淀粉结晶结构分为 $\mathrm{A}$-型、 $\mathrm{B}$-型和 $\mathrm{C}$-型 3 种 类型, 其中 $\mathrm{A}$-型晶体主要存在于禾谷类作物种子中, B-型晶体主要存在于植物块茎中和高直链作物种子 中, C-型晶体由 $\mathrm{A}$-型晶体和 $\mathrm{B}$-型晶体共同组成, 主 要存在于互类作物种子和薯蓣类根状茎中 ${ }^{[2]}$ 。

目前研究淀粉粒有序结构主要采用偏光显微镜 技术(polarized light microscopy, PLM)、粉末 X-射线 衍射技术(X-ray powder diffraction, XRD)、 ${ }^{13} \mathrm{C} \mathrm{CP} /$ MAS 固体核磁共振技术(cross-polarization magic-angle spinning nuclear magnetic resonance, ${ }^{13} \mathrm{C}$ CP/MAS NMR) 和傅里叶变换红外光谱技术(Fourier transform infrared spectroscopy, FTIR $)^{[2-5]}$ 。PLM 主要用于淀粉 粒晶体结构存在有无的定性分析 ${ }^{[3]}$ 。XRD 检测淀粉 分子的长程有序结构(即晶体), 虽然可用于淀粉粒 晶体类型的定性分析, 但在定量计算淀粉等多晶体 系的结晶度方面存在一定的困难 ${ }^{[2]}{ }^{13} \mathrm{C}$ CP/MAS NMR 测定淀粉分子的短程有序, 能够研究淀粉粒 的晶体类型和计算淀粉的结晶度和双螺旋含量, 但 仪器较昂贵, 测试样品用时较长, 结晶度和双螺旋 含量定量计算也非常复杂 ${ }^{[4]}$ 。FTIR 对淀粉结晶、分 子链的构象及螺旋结构的改变十分敏感, 用于淀粉 粒短程有序结构的研究 ${ }^{[5]}$ 。与 XRD 和 ${ }^{13} \mathrm{C}$ CP/MAS NMR 相比, FTIR 测试所需样品量极少 $(10 \mathrm{mg})$ 、操 作快速简单(2 3 min)、灵敏度高, 在国外已广泛用 于淀粉粒有序结构的分析 ${ }^{[5-7]}$, 但在国内这方面的 报道较少。

FTIR 分析淀粉粒结构时需要对原始波谱进行 去卷积, 其设置条件包括半峰宽和增强因子。半峰 宽设置从 $15 \mathrm{~cm}^{-1}$ 到 $26 \mathrm{~cm}^{-1}$, 增强因子设置从 1.5 到 2.6 都有文献报道 ${ }^{[5-8]}$, 如 Chung 等 ${ }^{[6]}$ 将半峰宽设为 $15 \mathrm{~cm}^{-1}$, 增强因子设为 1.5 ; Sevenou 等 ${ }^{[5]}$ 将半峰宽设 为 $19 \mathrm{~cm}^{-1}$, 增强因子设为 1.9 ; Rubens 等 ${ }^{[7]}$ 将半峰宽 设为 $21 \mathrm{~cm}^{-1}$, 增强因子设为 $1.7 ; \mathrm{Li}$ 等 ${ }^{[8]}$ 将半峰宽设
为 $26 \mathrm{~cm}^{-1}$ ，增强因子设为 2.4 。不同的设置条件，对 研究结果影响很大, 导致不同的文献结果没有可比 性, 但设置条件对研究结果到底有什么影响目前还 未见文献报道。FTIR 分析淀粉粒有序结构有 2 种模 式: 衰减全反射模式(attenuated total reflectance, ATR) 和透射模式(transmittance), 前者分析淀粉粒表层 2 $\mu \mathrm{m}$ 厚的结构信息，而后者分析的是整个淀粉粒的 结构信息 ${ }^{[5]}$ 。FTIR 衰减全反射和透射模式的原理和 制样方法完全不同, 但对研究结果有什么影响目前 也末见报道。FTIR 波谱的(1045/1022) $\mathrm{cm}^{-1}$ 和(1022/ 995) $\mathrm{cm}^{-1}$ 峰强度比值被看作是淀粉粒有序结构的指 标, 其中(1045/1022) $\mathrm{cm}^{-1}$ 峰强度比值反映淀粉分子 的有序程度, 其比值越大, 有序度越高 ${ }^{[5]}$ 。

本研究选用 $\mathrm{A}$-型晶体水稻淀粉、 $\mathrm{B}$-型晶体马铃 薯淀粉和 C-型晶体山药和晼豆淀粉为试验材料, 探 讨不同去卷积设置条件对淀粉 FTIR 分析结果的影 响, 并分析了 FTIR 在淀粉粒有序结构分析中的应用, 研究结果可为应用 FTIR 技术分析淀粉粒有序结构 提供重要的参考作用。

\section{1 材料与方法}

\section{1 试验材料}

水稻(Oryza sativa L.)品种特青(Teqing)、南京 11 (Nanjing 11)、武运粳 8 号(Wuyujing 8)、中花 11 (Zhonghua 11)、黄华占(Huanghuazhan)、桂花黄(Guihuahuang)、南特号(Nantehao)和桂朝 2 号(Guichao 2) 成熟种子采收于扬州大学试验田, 马铃薯(Solanum tuberosum L.)块茎、山药(Dioscorea opposita Thunb) 根状茎和踠豆(Pisum sativum L.) 种子采购于扬州市 农贸市场。

\section{2 天然淀粉粒的分离}

水稻精米和晼豆种子用 $\mathrm{ddH}_{2} \mathrm{O}$ 浸泡过夜, 马铃 薯块茎和山药根状茎去皮切成薄片。以研钵充分研 磨, 加 $\mathrm{ddH}_{2} \mathrm{O}$ 搅拌, 用 4 层纱布过滤, 然后将滤液分 别过 100、200、300 和 400 目篮, 将滤液 $3000 \times g$ 离 心 $10 \mathrm{~min}$, 获得沉淀。用 $\mathrm{dd}_{2} \mathrm{O}$ 悬浮沉淀, 再离心。 反复几次, 直到上清液澄清为止。沉淀经无水乙醇 脱水 2 次, $40^{\circ} \mathrm{C}$ 干燥 $2 \mathrm{~d}$, 研磨成粉末, 过 100 目篮, $-20^{\circ} \mathrm{C}$ 保存备用。

\section{3 酶解淀粉的制备}

参考 $\mathrm{Li}$ 等方法 ${ }^{[9]}$, 利用淀粉葡糖苷酶(amyloglucosidase, AMG)水解水稻特青淀粉。200 mg 淀粉悬 浮于 $40 \mathrm{~mL}$ 酶液 $\left(0.05 \mathrm{~mol} \mathrm{~L}^{-1}\right.$ 醋酸钠缓冲液, $\mathrm{pH} 4.5$, 
$100 \mathrm{U}$ AMG), 其中 AMG 来源于黑曲霉(Aspergillus niger)(Sigma-Aldrich 产品)。淀粉酶液在恒温振荡水 浴中, $100 \mathrm{r} \mathrm{min}^{-1} 、 55^{\circ} \mathrm{C}$ 条件下进行酶解反应。酶解 结束后, $3000 \times g$ 离心 $10 \mathrm{~min}$, 获得沉淀。用 $\mathrm{ddH}_{2} \mathrm{O}$ 悬浮沉淀, 再离心。重复 2 次, 沉淀经无水乙醇脱水 2 次, $40^{\circ} \mathrm{C}$ 干燥 $2 \mathrm{~d}$, 研磨成粉末, 过 100 目篮, $-20^{\circ} \mathrm{C}$ 保存备用。

\section{4 酸解淀粉的制备}

参考 Wei 等 ${ }^{[10]}$ 方法制备酸解淀粉。2 g 踠豆淀粉 加 $100 \mathrm{~mL} 2.2 \mathrm{~mol} \mathrm{~L}^{-1}$ 的 $\mathrm{HCl}$ 溶液, 于 $35^{\circ} \mathrm{C}$ 酸水解, 期间每天早晚摇匀淀粉沉淀 2 次。淀粉盐酸溶液经 $3000 \times g$ 离心 $10 \mathrm{~min}$, 用 $\mathrm{ddH}_{2} \mathrm{O}$ 悬浮沉淀, 再离心。 重复 3 次, 最后用无水乙醇脱水 2 次, $40^{\circ} \mathrm{C}$ 干燥 $2 \mathrm{~d}$, 研磨成粉末, 过 100 目篮, $-20^{\circ} \mathrm{C}$ 保存备用。

\section{5 直链淀粉含量测定}

参考 Konik-Rose 等 ${ }^{[11]}$ 方法并略有改进, 测定分 离淀粉粒的直链淀粉含量。将 $10 \mathrm{mg}$ 淀粉悬浮在 5 $\mathrm{mL}$ 尿素-二甲基亚砜溶液(UDMSO)(9 份 DMSO 和 1 份 $6 \mathrm{~mol} \mathrm{~L}^{-1}$ 尿素)中, 在 $95^{\circ} \mathrm{C}$ 水浴中溶解 $1 \mathrm{~h}$, 期间 间歇摇匀。 $1 \mathrm{~mL}$ 淀粉 UDMSO 溶液和 $1 \mathrm{~mL} 0.2 \% \mathrm{I}_{2}$ 、 $2 \% \mathrm{KI}(W / V)$ 染液用 $\mathrm{ddH}_{2} \mathrm{O}$ 定容到 $50 \mathrm{~mL}$ 。在分光光 度计上测定 $620 \mathrm{~nm}$ 波长下的吸光度。用马铃薯直链 淀粉和玉米支链淀粉按不同比例混合配制的标准溶 液制备标准曲线, 计算直链淀粉含量。每一样品做 3 次重复。

\section{6 透射 FTIR 光谱的采集}

淀粉经 $105^{\circ} \mathrm{C}$ 干燥 $2 \mathrm{~h}$, 采用 $\mathrm{KBr}$ 压片法, 按照 $1 \%$ 的比例与 $\mathrm{KBr}$ 充分混合、研磨、压片, 于红外光 谱仪(Varian 7000)上测试。扫描波数范围为 4000 $800 \mathrm{~cm}^{-1}$, 分辨率为 $4 \mathrm{~cm}^{-1}$, 采用 DTGS 检测器, 以 不含淀粉样品的 $\mathrm{KBr}$ 压片为空白扫背景, 扫描累加 64 次, 得到样品的红外光谱图。

\subsection{ATR-FTIR 光谱的采集}

将含有一定水分的淀粉, 加到 OMNI 采样器中, 利用 ATR 支架, 对淀粉样品进行全反射光谱扫描, 扫描设置同 1.6, 以水为空白扫背景。

\subsection{FTIR 波谱分析}

利用 FTIR 附带的软件分析波谱。首先选取波数 为 $1200 \sim 800 \mathrm{~cm}^{-1}$ 区域的谱图, 调整基线, 设置半峰 宽和增强因子, 对选取的波谱进行去卷积, 获得去 卷积波谱。利用 Origin 6.0 对波谱作图, 测定 1045、 1022 和 $995 \mathrm{~cm}^{-1}$ 各峰顶点到基线的高度(即峰强度), 计算(1045/1022) $\mathrm{cm}^{-1}$ 和(1022/995) $\mathrm{cm}^{-1}$ 的峰强度比值。

\section{2 结果与分析}

\section{1 淀粉 FTIR 波谱去卷积设置分析}

水稻品种特青含水量 70\%淀粉的 ATR-FTIR 和 干燥淀粉的透射 FTIR 原始波谱见图 1-a。参考相关 文献 ${ }^{[5-8]}$, 将半峰宽分别设置为 $15 、 19 、 23$ 和 $27 \mathrm{~cm}^{-1}$, 增强因子分别设置为 $1.5 、 1.9 、 2.3$ 和 2.7 , 对原始波 谱进行去卷积, 结果见图 1-b e。半峰宽和增强因子 不同, 对图谱各峰影响较大。(1045/1022) $\mathrm{cm}^{-1}$ 和 (1022/995) $\mathrm{cm}^{-1}$ 峰强度比值被看作是淀粉有序结构 的指标, 其中 (1045/1022) $\mathrm{cm}^{-1}$ 峰强度比值表示淀粉 分子结构中的有序结构和无定形结构的比例关系, 其比值越大, 颗粒内有序度越高 ${ }^{[5,12-13]}$ 。从图 1 可以 看出, 半峰宽为 $23 \mathrm{~cm}^{-1}$ 和 $27 \mathrm{~cm}^{-1}$, 增强因子为 1.5 时, 不能将 $1022 \mathrm{~cm}^{-1}$ 和 $995 \mathrm{~cm}^{-1}$ 附近的峰分开; 而 增强因子为 2.7 时, 会有许多背景峰出现。不同半峰 宽和增强因子设置条件下, ATR-FTIR 和透射 FTIR 去卷积波谱的(1045/1022) $\mathrm{cm}^{-1}$ 和(1022/995) $\mathrm{cm}^{-1}$ 峰 强度比值见表 1, 不同半峰宽和增强因子对 (1045/ 1022) $\mathrm{cm}^{-1}$ 和(1022/995) $\mathrm{cm}^{-1}$ 峰强度比值影响较大。 综合分析, 选择半峰宽为 $19 \mathrm{~cm}^{-1}$, 增强因子为 1.9 时, 对 FTIR 原始波谱去卷积效果较好。比较透射 FTIR 波谱和 ATR-FTIR 波谱的原始波谱和去卷积波 谱, 以及(1045/1022) $\mathrm{cm}^{-1}$ 和(1022/995) $\mathrm{cm}^{-1}$ 峰强度 的比值, 可以看出, 透射模式和 ATR 模式对淀粉的 分析结果相差较大。

\section{2 不同晶体类型 FTIR 波谱分析}

水稻、马铃薯和山药淀粉分别代表 A-型、B-型 和 C-型晶体淀粉, 含水量 70\%淀粉的 ATR-FTIR 原 始波谱见图 2-A, 干燥淀粉的透射 FTIR 原始波谱见 图 2-B。不同晶体类型之间原始波谱的主要区别发 生在 1050 950 $\mathrm{cm}^{-1}$, ATR-FTIR 与透射 FTIR 原始波 谱之间差别较大。对原始波谱进行去卷积, 半峰宽 设为 $19 \mathrm{~cm}^{-1}$ 和增强因子设为 1.9 , 去卷积的 ATRFTIR 波谱和透射 FTIR 波谱分别见图 2-C 和 2-D, (1045/1022) $\mathrm{cm}^{-1}$ 和(1022/995) $\mathrm{cm}^{-1}$ 峰强度的比值见 表 2。在 ATR-FTIR 波谱中, 水稻淀粉的去卷积波谱 明显不同于马铃薯和山药, 水稻淀粉 (1045/1022) $\mathrm{cm}^{-1}$ 峰强度比值明显比马铃薯和山药低, (1022/995) $\mathrm{cm}^{-1}$ 峰强度比值明显比马铃薯和山药高, 而马铃薯 和山药淀粉的(1045/1022) $\mathrm{cm}^{-1}$ 和(1022/995) $\mathrm{cm}^{-1}$ 峰 强度比值相似。在透射 FTIR 波谱中, 山药淀粉 (1045/1022) $\mathrm{cm}^{-1}$ 峰强度比值比水稻和马铃薯高, $(1022 / 995) \mathrm{cm}^{-1}$ 峰强度比值明显比水稻和马铃薯低, 
而水稻和马铃薯淀粉的(1045/1022) $\mathrm{cm}^{-1}$ 和(1022/995) $\mathrm{cm}^{-1}$ 峰强度比值相似。ATR-FTIR 和透射 FTIR 波谱 间的比较表明, 透射模式下(1022/995) $\mathrm{cm}^{-1}$ 峰强度 比值明显比 ATR 模式下高, 水稻和山药淀粉(1045/

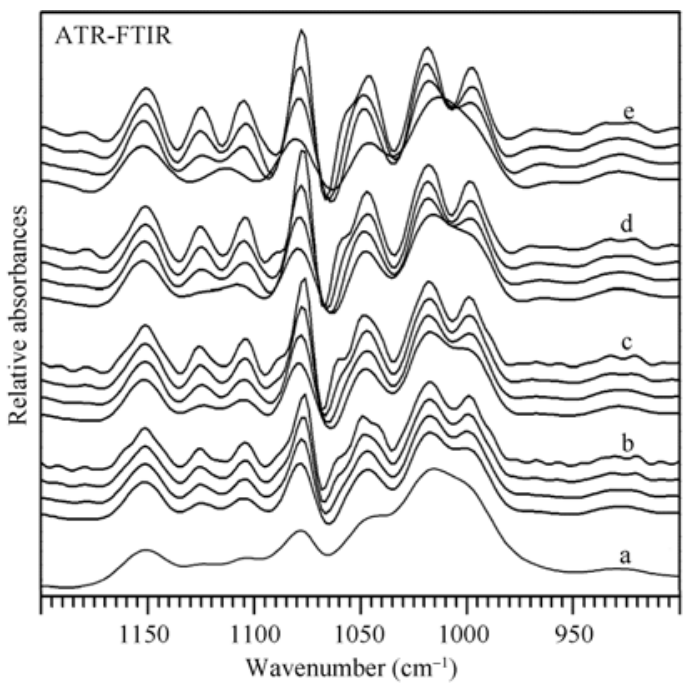

1022) $\mathrm{cm}^{-1}$ 峰强度比值相似, 而马铃薯淀粉(1045/ 1022) $\mathrm{cm}^{-1}$ 峰强度比值在透射模式下比 ATR 模式下 低。上述结果表明, 淀粉晶体类型不同, ATR-FTIR 和透射 FTIR 波谱表现也不同。

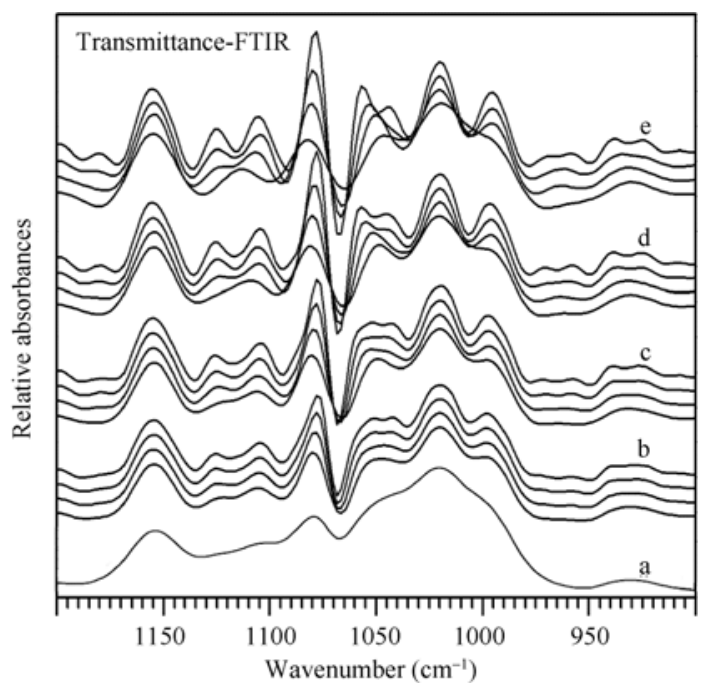

图 1 水稻淀粉 FTIR 原始波谱和去卷积波谱

Fig. 1 Original and deconvoluted FTIR spectra of rice starches

a：原始波谱; b e: 去卷积波谱，半峰宽分别为 15 (b)、19 (c)、23 (d) 和 $27 \mathrm{~cm}^{-1}$ (e), 其中每个半峰宽的增强因子从下至上分别为 $1.5 、 1.9 、 2.3$ 和 2.7 。

a: Original spectrum; b-e: Deconvoluted spectra, the peak half-width of wavenumber was 15 (b), 19 (c), 23 (d), and $27 \mathrm{~cm}^{-1}$ (e), respectively. The resolution enhancement factor of the deconvoluted spectrum is 1.5, 1.9, 2.3, and 2.7 from the bottom to the top in each peak half-width of wavenumber.

表 1 水稻淀粉 FTIR 去卷积波谱的(1045/1022) $\mathrm{cm}^{-1}$ 和(1022/995) $\mathrm{cm}^{-1}$ 峰强度比值比较

Table 1 IR ratio of the absorbances $(1045 / 1022) \mathrm{cm}^{-1}$ and $(1022 / 995) \mathrm{cm}^{-1}$ for deconvoluted FTIR spectra of rice starches

\begin{tabular}{|c|c|c|c|c|c|}
\hline \multirow{2}{*}{$\begin{array}{c}\text { 半峰宽 } \\
\text { Peak half-width of } \\
\text { wavenumber }\left(\mathrm{cm}^{-1}\right) \\
\end{array}$} & \multirow{2}{*}{$\begin{array}{c}\text { 增强因子 } \\
\text { Resolution enhancement } \\
\text { factor } \\
\end{array}$} & \multicolumn{2}{|c|}{ 衰减全反射 FTIR ATR-FTIR } & \multicolumn{2}{|c|}{ 透射 FTIR Transmittance-FTIR } \\
\hline & & $(1045 / 1022) \mathrm{cm}^{-1}$ & $(1022 / 995) \mathrm{cm}^{-1}$ & $(1045 / 1022) \mathrm{cm}^{-1}$ & $(1022 / 995) \mathrm{cm}^{-1}$ \\
\hline \multirow{4}{*}{15} & 1.5 & 0.54 & 1.24 & 0.63 & 1.54 \\
\hline & 1.9 & 0.57 & 1.23 & 0.62 & 1.55 \\
\hline & 2.3 & 0.57 & 1.22 & 0.61 & 1.51 \\
\hline & 2.7 & 0.58 & 1.20 & 0.62 & 1.50 \\
\hline \multirow{4}{*}{19} & 1.5 & 0.55 & 1.25 & 0.65 & 1.56 \\
\hline & 1.9 & 0.57 & 1.28 & 0.59 & 1.59 \\
\hline & 2.3 & 0.61 & 1.25 & 0.56 & 1.57 \\
\hline & 2.7 & 0.59 & 1.23 & 0.55 & 1.55 \\
\hline \multirow{4}{*}{23} & 1.5 & 0.55 & 1.29 & 0.67 & 1.58 \\
\hline & 1.9 & 0.59 & 1.33 & 0.65 & 1.61 \\
\hline & 2.3 & 0.60 & 1.31 & 0.51 & 1.58 \\
\hline & 2.7 & 0.67 & 1.26 & 0.52 & 1.55 \\
\hline \multirow{4}{*}{27} & 1.5 & $\mathrm{nd}^{\mathrm{a}}$ & $\mathrm{nd}^{\mathrm{a}}$ & 0.64 & 1.64 \\
\hline & 1.9 & 0.65 & 1.37 & 0.71 & 1.65 \\
\hline & 2.3 & 0.61 & 1.40 & 0.66 & 1.64 \\
\hline & 2.7 & 0.64 & 1.34 & 0.45 & 1.59 \\
\hline
\end{tabular}

${ }^{\mathrm{a}}$ nd: 未检测。 ${ }^{\mathrm{a}}$ nd: not detected. 

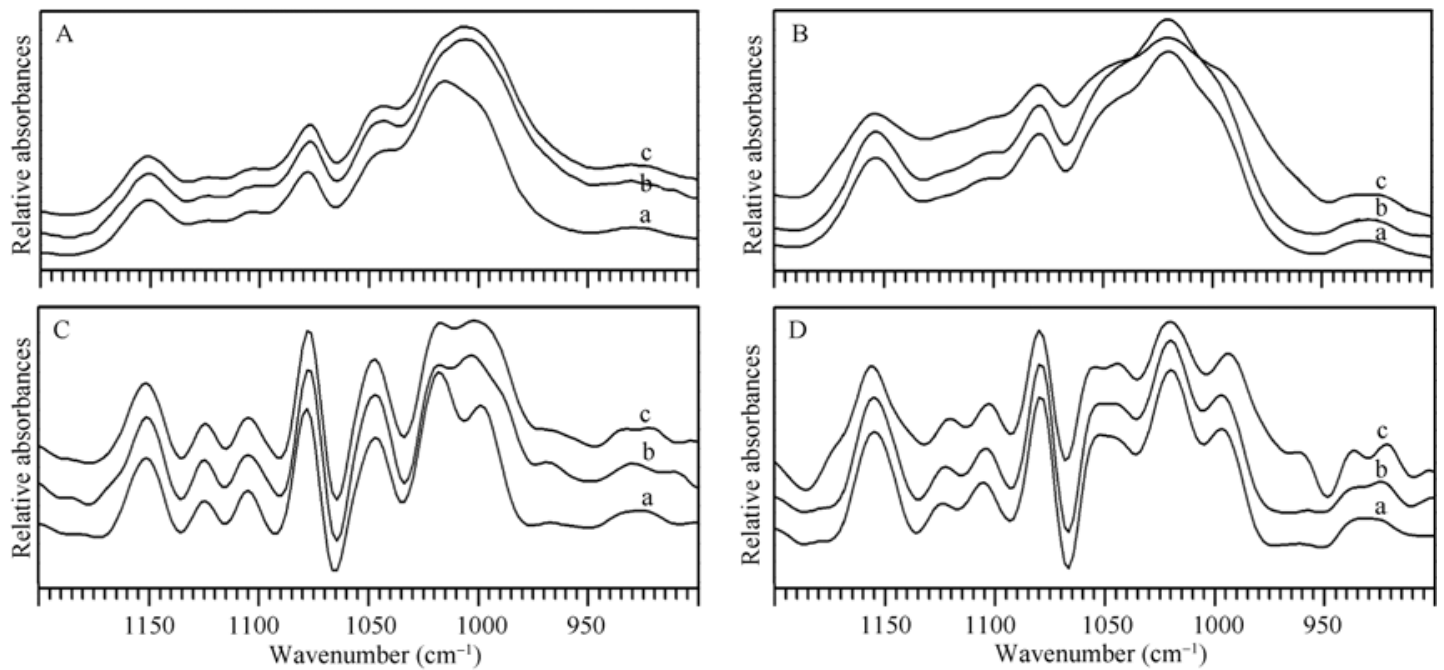

图 2 不同晶体类型淀粉 FTIR 原始波谱和去卷积波谱

Fig. 2 Original and deconvoluted FTIR spectra of starches with different crystalline types A: ATR-FTIR 原始波谱; B：透射 FTIR 原始波谱; C：去卷积 ATR-FTIR 波谱; D：去卷积透射 FTIR 波谱; a：水稻; b: 马铃薯; c：山药。

A, B: original spectra; C, D: deconvoluted spectra; A, C: ATR-FTIR; B, D: transmittance-FTIR; a: rice; b: potato; c: Chinese yam.

表 2 不同晶体类型淀粉 FTIR 去卷积波谱的(1045/1022) $\mathrm{cm}^{-1}$ 和 $(1022 / 995) \mathrm{cm}^{-1}$ 峰强度比值比较

Table 2 IR ratio of the absorbances $(1045 / 1022) \mathrm{cm}^{-1}$ and $(1022 / 995) \mathrm{cm}^{-1}$ for deconvoluted FTIR spectra of starches with different crystalline types

\begin{tabular}{|c|c|c|c|c|}
\hline \multirow{2}{*}{$\begin{array}{c}\text { 试验材料 } \\
\text { Plant material }\end{array}$} & \multicolumn{2}{|c|}{ 衰减全反射 FTIRＡTR-FTIR } & \multicolumn{2}{|c|}{ 透射 FTIR Transmittance-FTIR } \\
\hline & $(1045 / 1022) \mathrm{cm}^{-1}$ & $(1022 / 995) \mathrm{cm}^{-1}$ & $(1045 / 1022) \mathrm{cm}^{-1}$ & $(1022 / 995) \mathrm{cm}^{-1}$ \\
\hline 水稻 Rice & 0.57 & 1.28 & 0.59 & 1.59 \\
\hline 马铃薯 Potato & 0.75 & 0.94 & 0.60 & 1.54 \\
\hline 山药 Chinese yam & 0.71 & 0.97 & 0.71 & 1.28 \\
\hline
\end{tabular}

\section{3 含水量对淀粉 ATR-FTIR 波谱影响分析}

应用 ATR-FTIR 分析天然淀粉的有序结构, 样 品含水量对分析结果会产生影响。对不同含水量水 稻特青淀粉进行 ATR-FTIR 分析, 将半峰宽设为 19 $\mathrm{cm}^{-1}$ 和增强因子设为 1.9, 去卷积的 ATR-FTIR 波谱 见图 3, (1045/1022) $\mathrm{cm}^{-1}$ 和(1022/995) $\mathrm{cm}^{-1}$ 峰强度比 值见表 3。从图谱中可以看出, 水分含量对波谱各峰 强度有影响。样品含水量超过 $60 \%$ 时, 对波谱基本 没有影响。因此，对淀粉进行 ATR-FTIR 波谱分析时， 样品含水量应控制在 $60 \%$ 以上, 以便于样品间的比 较, 排除水分的干扰。

\section{4 酸解淀粉 ATR-FTIR 波谱分析}

踠互淀粉经盐酸水解, 对不同水解天数含水量 70\%淀粉的 ATR-FTIR 原始波谱进行去卷积, 半峰宽 设为 $19 \mathrm{~cm}^{-1}$ 和增强因子设为 1.9 的去卷积波谱见图 4, (1045/1022) $\mathrm{cm}^{-1}$ 和(1022/995) $\mathrm{cm}^{-1}$ 峰强度比值见 表 4。从图谱和峰强度比值可以看出, 伴随酸解, $1022 \mathrm{~cm}^{-1}$ 峰强度逐渐降低, (1045/1022) $\mathrm{cm}^{-1}$ 峰强度 比值逐渐升高, 而(1022/995) $\mathrm{cm}^{-1}$ 峰强度比值逐渐
降低, 表明酸水解淀粉无定形结构成分比有序结构 成分快，导致淀粉有序度提高。

\section{5 酶解淀粉 FTIR 波谱分析}

利用 AMG 对水稻特青淀粉进行酶水解，对酶 解含水量 70\%淀粉的 ATR-FTIR 和干燥淀粉的透射 FTIR 原始波谱进行去卷积, 半峰宽设为 $19 \mathrm{~cm}^{-1}$ 和 增强因子设为 1.9 的去卷积波谱见图 5, (1045/1022) $\mathrm{cm}^{-1}$ 和(1022/995) $\mathrm{cm}^{-1}$ 峰强度比值见表 5。从图谱和 峰强度比值可以看出, ATR-FTIR 和透射 FTIR 分析 结果完全不同。伴随酶解, ATR-FTIR 获得的(1045/ 1022) $\mathrm{cm}^{-1}$ 峰强度比值变化不大, 透射 FTIR 获得的 (1045/1022) $\mathrm{cm}^{-1}$ 峰强度比值显著增加, 表明 AMG 水解淀粉对淀粉外部区域的有序度影响不大，但明 显提高整个淀粉的有序度。

\section{6 不同直链淀粉含量稻米淀粉 ATR-FTIR 波} 谱分析

直链淀粉含量是淀粉理化特性最重要的指标, 决定淀粉的品质和用途。对含水量 $70 \%$ 的不同直链 淀粉含量的稻米淀粉进行 ATR-FTIR 分析, 半峰宽 


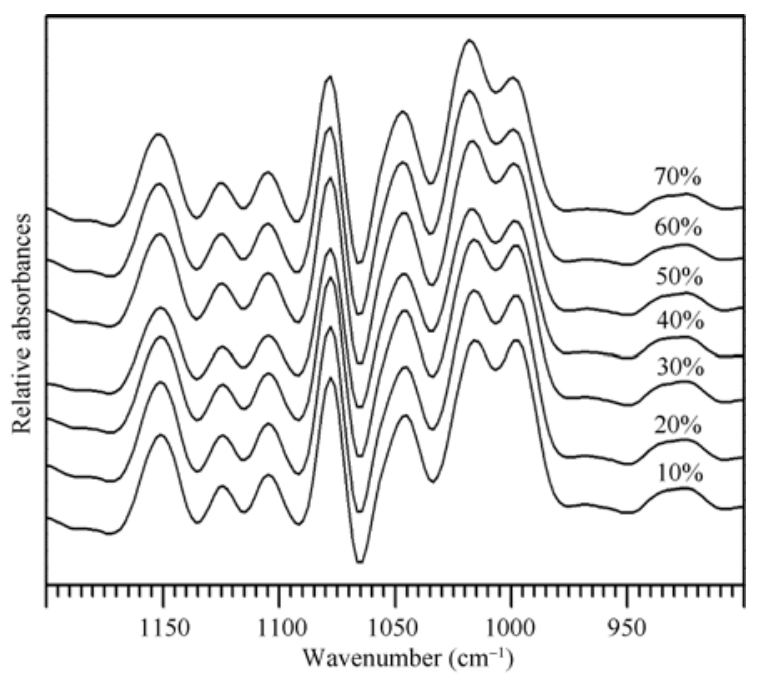

图 3 不同含水量水稻淀粉 ATR-FTIR 去卷积波谱

Fig. 3 Deconvoluted ATR-FTIR spectra of rice starches with different water contents

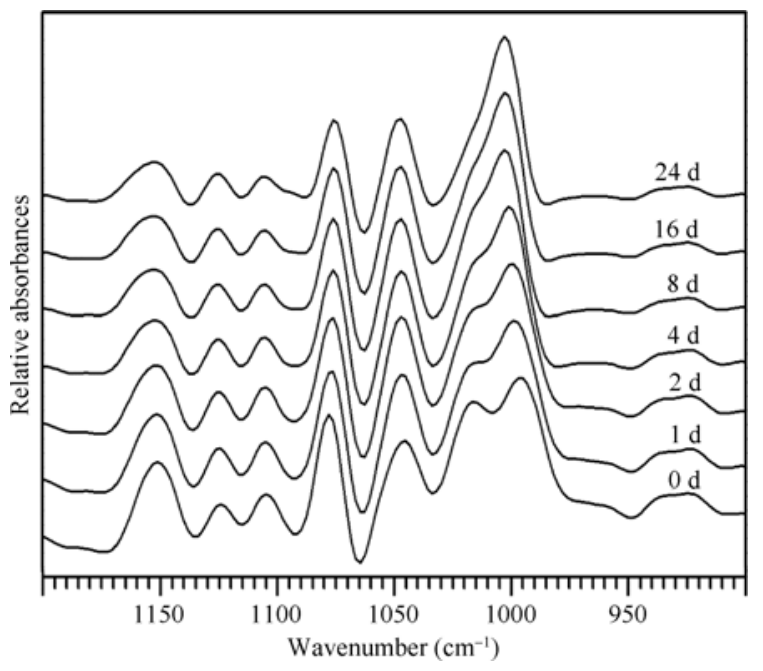

图 4 酸水解豌豆淀粉 ATR-FTIR 去卷积波谱

Fig. 4 Deconvoluted ATR-FTIR spectra of pea starches by acid hydrolysis

表 3 不同含水量水稻淀粉 ATR-FTIR 去卷积波谱的 $(1045 / 1022) \mathrm{cm}^{-1}$ 和 $(1022 / 995) \mathrm{cm}^{-1}$ 峰强度比值比较

Table 3 IR ratio of the absorbances $(1045 / 1022) \mathrm{cm}^{-1}$ and $(1022 / 995) \mathrm{cm}^{-1}$ for deconvoluted ATR-FTIR spectra of rice starches with different water contents

\begin{tabular}{|c|c|c|}
\hline \multirow{2}{*}{$\begin{array}{c}\text { 水分含量 } \\
\text { Water content }\end{array}$} & 衰减全反射 FTIR & ATR-FTIR \\
\hline & $(1045 / 1022) \mathrm{cm}^{-1}$ & $(1022 / 995) \mathrm{cm}^{-1}$ \\
\hline $10 \%$ & 0.58 & 1.00 \\
\hline $20 \%$ & 0.59 & 1.03 \\
\hline $30 \%$ & 0.60 & 1.03 \\
\hline $40 \%$ & 0.62 & 1.08 \\
\hline $50 \%$ & 0.62 & 1.16 \\
\hline $60 \%$ & 0.62 & 1.28 \\
\hline $70 \%$ & 0.62 & 1.29 \\
\hline
\end{tabular}

表 4 酸水解腕豆淀粉 ATR-FTIR 去卷积波谱的(1045/1022) $\mathrm{cm}^{-1}$ 和(1022/995) $\mathrm{cm}^{-1}$ 峰强度比值比较

Table 4 IR ratio of the absorbances $(1045 / 1022) \mathrm{cm}^{-1}$ and $(1022 / 995) \mathrm{cm}^{-1}$ for deconvoluted ATR-FTIR spectra of acid hydrolyzed pea starches

\begin{tabular}{ccc}
\hline $\begin{array}{c}\text { 水解时间 } \\
\text { Hydrolysis time }\end{array}$ & \begin{tabular}{c} 
衰减全反射 FTIR \\
\cline { 2 - 3 }$(1045 / 1022) \mathrm{cm}^{-1}$
\end{tabular} & $\begin{array}{c}\text { ATR-FTIR } \\
(1022 / 995) \mathrm{cm}^{-1}\end{array}$ \\
\hline $0 \mathrm{~d}$ & 0.70 & 0.85 \\
$1 \mathrm{~d}$ & 0.88 & 0.74 \\
$2 \mathrm{~d}$ & 0.90 & 0.72 \\
$4 \mathrm{~d}$ & 0.90 & 0.66 \\
$8 \mathrm{~d}$ & 0.93 & 0.64 \\
$16 \mathrm{~d}$ & 0.96 & 0.56 \\
$24 \mathrm{~d}$ & 1.07 & 0.45 \\
\hline
\end{tabular}

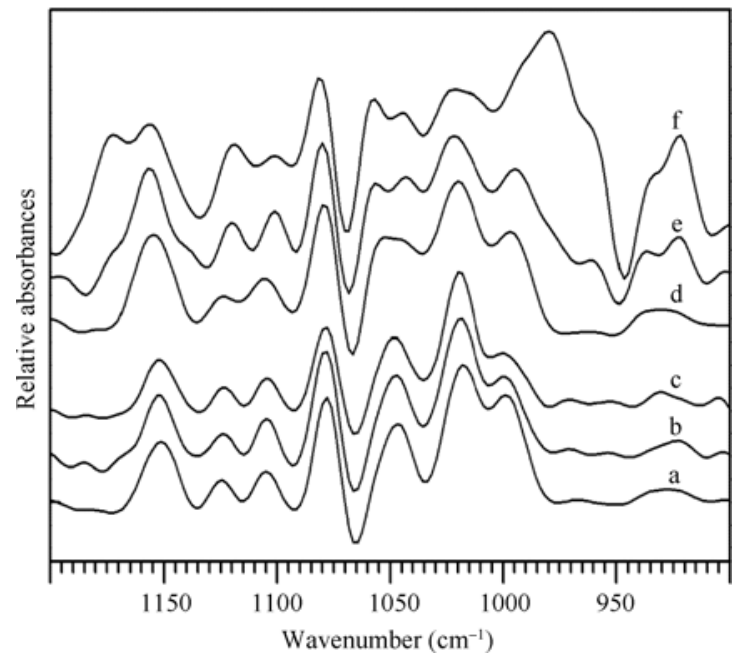

图 5 葡糖苷酶水解水稻淀粉 FTIR 去卷积波谱

Fig. 5 Deconvoluted FTIR spectra of amyloglucosidase hydrolyzed rice starches

a C: ATR-FTIR 波谱; d f: 透射 FTIR 波谱; $\mathrm{a}, \mathrm{d}$ : 葡糖苷酶水解 0 $\mathrm{h}$ 淀粉; b 和 e: 葡糖苷酶水解 $1.5 \mathrm{~h}$ 淀粉; c, f: 葡糖苷酶水解 $12 \mathrm{~h}$ 淀粉。

a-c: ATR-FTIR spectra; d-f: Transmittance-FTIR spectra; a-f: Hydrolyzed starches by amyloglucosidase for $0 \mathrm{~h}(\mathrm{a}, \mathrm{d})$, $1.5 \mathrm{~h}(\mathrm{~b}, \mathrm{e})$, and $12 \mathrm{~h}$ (c, f).

设为 $19 \mathrm{~cm}^{-1}$ 和增强因子设为 1.9 的去卷积波谱见图 6, (1045/1022) $\mathrm{cm}^{-1}$ 和(1022/995) $\mathrm{cm}^{-1}$ 峰强度比值见 表 6。从图谱和峰强度比值可以看出, 直链淀粉含量 不同的稻米淀粉 ATR-FTIR 波谱相似，表明直链淀 粉对淀粉粒表层有序结构没有影响。

\section{3 讨论}

FTIR 波谱在 1200 900 $\mathrm{cm}^{-1}$ 区域有 8 个红外吸 收峰, 其中 1150、1124 和 $1103 \mathrm{~cm}^{-1}$ 附近的吸收峰 为 C-O-C 的伸缩振动和 C-C、 C-H 的骨架振动; 1080 $\mathrm{cm}^{-1}$ 附近的吸收峰是 C-O 的伸缩振动和 C-C 的骨架 


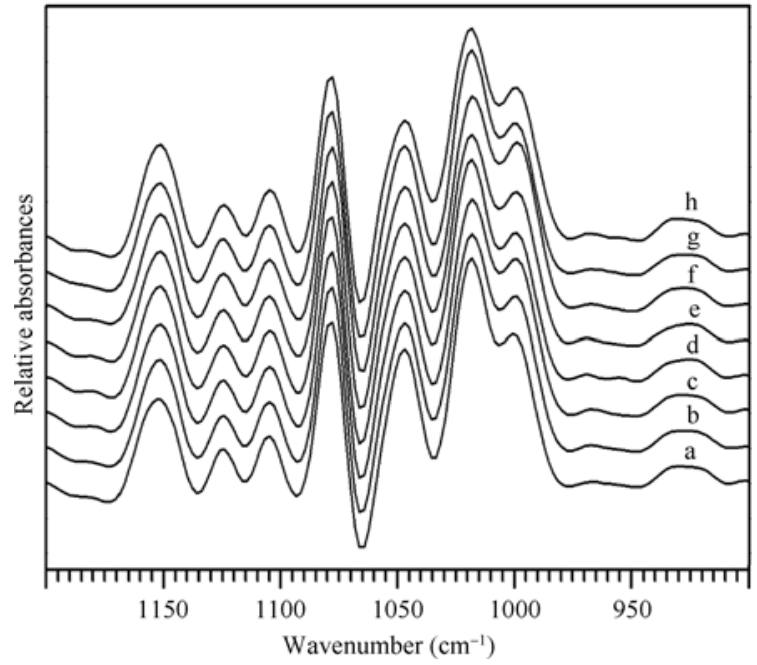

图 6 不同直链淀粉含量水稻淀粉 ATR-FTIR 去卷积波谱

Fig. 6 Deconvoluted ATR-FTIR spectra of rice starches with different amylose content

a: 南京 11; b: 武运粳 8 号; c: 中花 11; d: 黄华占; e: 桂花黄;

f: 南特号; g: 特青; h: 桂朝 2 号。

a: Nanjing 11; b: Wuyunjing 8; c: Zhonghua 11; d: Huanghuazhan; e: Guihuahuang; f: Nantehao; g: Teqing; h: Guichao 2.

振动的复合表现; $1045 \mathrm{~cm}^{-1}$ 附近的吸收峰是淀粉结 晶区的结构特征, 对应于淀粉聚集态结构中的有序 结构; $1022 \mathrm{~cm}^{-1}$ 附近的吸收峰则是淀粉非晶区的结 构特征, 对应于淀粉大分子的无规线团结构; 995 $\mathrm{cm}^{-1}$ 附近的吸收峰主要是由于 $\mathrm{C}-\mathrm{OH}$ 的弯曲振动引
起的, 对应于淀粉大分子的羟基间所形成的氢键结 构; $930 \mathrm{~cm}^{-1}$ 附近的吸收峰则是葡萄糖环的振动吸 收峰 ${ }^{[5,12-13]}$ 。虽然不同种类的淀粉红外吸收峰的位置 并无差别, 但吸收峰的强度在不同淀粉间有差异。 (1045/1022) $\mathrm{cm}^{-1}$ 和(1022/995) $\mathrm{cm}^{-1}$ 峰强度比值被看 作是淀粉有序结构的指标, 其中(1045/1022) $\mathrm{cm}^{-1}$ 峰 强度比值反映淀粉分子的有序程度, 其比值越大, 有序度越高 ${ }^{[5,12-13]}$ 。上述的 FTIR 波谱红外吸收峰的 识别和峰强度的计算不是直接来源于 FTIR 原始波 谱, 而是来源于去卷积后的波谱。本文的水稻、马 铃薯和山药淀粉的 ATR-FTIR 原始波谱和透射 FTIR 原始波谱在 1200 900 $\mathrm{cm}^{-1}$ 区域都不能完全区分相 对应的 8 个红外吸收峰, 有些吸收峰以肩峰的形式 出现(图 2-A, B), 这与前人的研究结果一致 ${ }^{[5-8]}$ 。对 FTIR 原始波谱去卷积要设置参数半峰宽和增强因 子, 本文将半峰宽分别设置为 $15 、 19 、 23$ 和 $27 \mathrm{~cm}^{-1}$, 增强因子分别设置为 $1.5 、 1.9 、 2.3$ 和 2.7 , 对原始波 谱进行去卷积(图 1), 去卷积波谱能够清晰识别红外 吸收峰和精确计算峰强度。从红外吸收峰和(1045/ 1022) $\mathrm{cm}^{-1} 、(1022 / 995) \mathrm{cm}^{-1}$ 峰强度比值可以看出, 不同的半峰宽和增强因子去卷积设置对波谱分析结 果影响较大。前人的研究中, 对 FTIR 波谱的去卷积 设置都不统一, 半峰宽从 $15 \mathrm{~cm}^{-1}$ 到 $26 \mathrm{~cm}^{-1}$, 增强

表 5 葡糖苷酶水解水稻淀粉 FTIR 去卷积波谱的 $(1045 / 1022) \mathrm{cm}^{-1}$ 和 $(1022 / 995) \mathrm{cm}^{-1}$ 峰强度比值比较

Table 5 IR ratio of the absorbances $(1045 / 1022) \mathrm{cm}^{-1}$ and $(1022 / 995) \mathrm{cm}^{-1}$ for deconvoluted FTIR spectra of amyloglucosidase hydrolyzed rice starches

\begin{tabular}{|c|c|c|c|c|}
\hline \multirow{2}{*}{$\begin{array}{c}\text { 水解时间 } \\
\text { Hydrolysis time }\end{array}$} & \multicolumn{2}{|c|}{ 衰减全反射 FTIR ATR-FTIR } & \multicolumn{2}{|c|}{ 透射 FTIR Transmittance-FTIR } \\
\hline & $(1045 / 1022) \mathrm{cm}^{-1}$ & $(1022 / 995) \mathrm{cm}^{-1}$ & $(1045 / 1022) \mathrm{cm}^{-1}$ & $(1022 / 995) \mathrm{cm}^{-1}$ \\
\hline $0 \mathrm{~h}$ & 0.57 & 1.28 & 0.59 & 1.59 \\
\hline $1.5 \mathrm{~h}$ & 0.58 & 1.75 & 0.71 & 1.30 \\
\hline $12 \mathrm{~h}$ & 0.52 & 2.42 & 0.86 & 0.94 \\
\hline
\end{tabular}

表 6 不同直链淀粉含量水稻淀粉 ATR-FTIR 去卷积波谱的(1045/1022) $\mathrm{cm}^{-1}$ 和(1022/995) $\mathrm{cm}^{-1}$ 峰强度比值比较

Table 6 IR ratio of the absorbances $(1045 / 1022) \mathrm{cm}^{-1}$ and $(1022 / 995) \mathrm{cm}^{-1}$ for deconvoluted ATR-FTIR spectra of rice starches with different amylose content

\begin{tabular}{|c|c|c|c|}
\hline \multirow{2}{*}{$\begin{array}{c}\text { 水稻品种 } \\
\text { Rice cultivar }\end{array}$} & \multirow{2}{*}{$\begin{array}{c}\text { 直链淀粉含量 } \\
\text { Amylose content (\%) }\end{array}$} & \multicolumn{2}{|c|}{ 衰减全反射 FTIR ATR-FTIR } \\
\hline & & $(1045 / 1022) \mathrm{cm}^{-1}$ & $(1022 / 995) \mathrm{cm}^{-1}$ \\
\hline 南京 11 Nanjing 11 & $10.6 \pm 0.3$ & 0.60 & 1.51 \\
\hline 武运粳 8 号 Wuyunjing 8 & $16.7 \pm 0.4$ & 0.57 & 1.49 \\
\hline 中花 11 Zhonghua 11 & $17.8 \pm 0.5$ & 0.58 & 1.41 \\
\hline 黄华占 Huanghuazhan & $19.1 \pm 0.4$ & 0.57 & 1.40 \\
\hline 桂花黄 Guihuahuang & $22.8 \pm 0.3$ & 0.55 & 1.51 \\
\hline 南特号 Nantehao & $27.6 \pm 0.5$ & 0.57 & 1.38 \\
\hline 特青 Teqing & $28.5 \pm 0.4$ & 0.57 & 1.28 \\
\hline 桂朝 2 号 Guichao 2 & $30.0 \pm 0.2$ & 0.56 & 1.41 \\
\hline
\end{tabular}


因子从 1.5 到 2.6 都有文献报道, 导致研究结果没有 可比性 ${ }^{[5-8]}$ 。参考相关文献, 结合本文的研究结果, 将半峰宽设为 $19 \mathrm{~cm}^{-1}$, 增强因子设为 1.9 , 对 FTIR 原始波谱去卷积效果较好。ATR-FTIR 分析的样品含 有水分, 淀粉水分含量影响其相对有序度 ${ }^{[5-8]}$ 。本文 的结果表明, 水分含量对波谱各峰强度有影响, 但 当样品含水量超过 $60 \%$ 时, 对波谱基本没有影响。 因此, 对淀粉进行 ATR-FTIR 波谱分析时, 样品含水 量应控制在 $60 \%$ 以上, 以便于样品间的比较, 排除 水分的干扰。

根据 ATR 原理, ATR-FTIR 波谱分析的是淀粉粒 表层 $2 \mu \mathrm{m}$ 厚的结构信息, 而 KBr 压片透射 FTIR 分 析的是整个淀粉粒的结构信息 ${ }^{[5]}$ 。比较透射 FTIR 波 谱和 ATR-FTIR 波谱的原始波谱和去卷积波谱, 以 及(1045/1022) $\mathrm{cm}^{-1}$ 和(1022/995) $\mathrm{cm}^{-1}$ 峰强度的比值, 可以看出, 透射模式和 ATR 模式对淀粉的分析结果 相差较大, 也即淀粉粒外部区域的有序结构与整个 淀粉粒的有序结构有差异。本文的结果表明, 水稻 和山药淀粉 ATR-FTIR 和透射 FTIR 波谱的(1045/ 1022) $\mathrm{cm}^{-1}$ 峰强度比值相似, 而马铃薯淀粉的(1045/ 1022) $\mathrm{cm}^{-1}$ 峰强度比值在 ATR-FTIR 波谱中明显比透 射 FTIR 波谱高, 表明水稻和山药淀粉外部区域和内 部区域的结构有序性相同, 而马铃薯淀粉外部区域 的结构有序性比内部高。马铃薯和山药淀粉的 ATRFTIR 波谱(1045/1022) $\mathrm{cm}^{-1}$ 峰强度比值比水稻高, 表明马铃薯和山药淀粉外部区域的结构有序性比水 稻高。

淀粉在加工利用过程中经常涉及到淀粉的酸水 解、酶水解和糊化, FTIR 可以用来研究淀粉加工利 用过程中有序结构的变化。研究表明, 酸水解降解 淀粉的无定形结构成分比有序结构成分快, 本文的 ATR-FTIR 波谱分析结果也表明, 啘豆淀粉随着酸 解时间的延长, $1022 \mathrm{~cm}^{-1}$ 无定形峰强度逐渐降低, (1045/1022) $\mathrm{cm}^{-1}$ 峰强度比值逐渐升高, 表明淀粉有 序度提高, 与前人在马铃薯、小麦和水稻淀粉酸水 解研究结果相一致 ${ }^{[5,14]}$ 。淀粉有序结构对水解酶具有 较高的抗性 ${ }^{[5]}$, 本文 ATR-FTIR 波谱分析表明，马铃 薯和山药淀粉在淀粉粒表层区域的有序度比水稻淀 粉高, 表明马铃薯和山药淀粉对酶水解具有较高的 抗性。伴随 AMG 酶解水稻淀粉, ATR-FTIR 波谱的 (1045/1022) $\mathrm{cm}^{-1}$ 峰强度比值变化不大, 而透射 FTIR 波谱的(1045/1022) $\mathrm{cm}^{-1}$ 峰强度比值显著增加, 表明 淀粉外部区域的有序性结构在酶解过程中没有明显
改变, 而淀粉粒内部的无定形成分被优先降解或降 解较快, 导致淀粉内部有序性结构比例增加。淀粉 在糊化过程中, 有序结构逐渐被破坏转变成无定形 结构成分, Sevenou 等 ${ }^{[5]}$ 和 Wei 等 ${ }^{[15]}$ 利用 FTIR 分析 了马铃薯和水稻淀粉糊化过程中有序结构的变化, ATR-FTIR 波谱的 $1022 \mathrm{~cm}^{-1}$ 无定形峰强度随着糊化 温度的升高而增强, (1045/1022) $\mathrm{cm}^{-1}$ 峰强度比值逐 渐降低，表明淀粉的有序度在不断降低。天然淀粉 有 A-型、B-型和 C-型晶体 3 种类型, 本文的结果表 明, 天然马铃薯、山药和水稻淀粉的 FTIR 波谱表现 明显不同。van Soest 等 ${ }^{[16]}$ 认为淀粉的 FTIR 波谱与 XRD 波谱具有相关性, Sevenou 等 ${ }^{[5]}$ 的结果却表明, 淀粉 FTIR 波谱与 XRD 波谱没有相关性。本文的结 果表明, 非天然淀粉(酸解、酶解或糊化等淀粉)FTIR 波谱与 XRD 波谱没有相关性, 但天然淀粉与 XRD 波谱具有相关性。直链淀粉含量是淀粉最重要的理 化特性之一, 决定淀粉的品质和用途。本文的结果 表明, 直链淀粉含量不同的稻米淀粉 ATR-FTIR 波 谱没有差异, (1045/1022) $\mathrm{cm}^{-1}$ 和(1022/995) $\mathrm{cm}^{-1}$ 峰 强度的比值也与直链淀粉含量没有相关性, 表明稻 米淀粉粒表层有序结构不受直链淀粉含量的影响。

\section{4 结论}

不同去卷积设置条件对 FTIR 波谱和相关峰强 度影响较大, 以半峰宽 $19 \mathrm{~cm}^{-1}$ 和增强因子 1.9 的设 置对 FTIR 原始波谱进行去卷积, 获得的结果较好。 天然淀粉晶体类型不同，其 FTIR 波谱也有差异。淀 粉中水分含量超过 $60 \%$ 时，水分含量对 ATR-FTIR 波谱没有影响。酸水解降解淀粉无定形结构成分比 有序结构成分快，提高淀粉的有序度。淀粉葡糖甘 酶水解淀粉对淀粉外部区域的有序度影响不大, 但 明显提高整个淀粉的有序度。稻米淀粉粒表层有序 结构不受直链淀粉含量的影响。上述研究结果为应 用 FTIR 分析淀粉加工利用过程中有序结构的变化 提供了重要的参考。

\section{References}

[1] Gallant D J, Bouchet B, Baldwin P M. Microscopy of starch: evidence of a new level of granule organization. Carbohydr Polym, 1997, 32: 177-191

[2] Cheetham N W H, Tao L. Variation in crystalline type with amylose content in maize starch granules: an X-ray powder diffraction study. Carbohydr Polym, 1998, 36: 277-284

[3] Wei C X, Qin F L, Zhu L J, Zhou W D, Chen Y F, Wang Y P, Gu M H, Liu Q Q. Microstructure and ultrastructure of high-amylose 
rice resistant starch granules modified by antisense RNA inhibition of starch branching enzyme. J Agric Food Chem, 2010, 58: 1224-1232

[4] Atichokudomchai N, Varavinit S, Chinachoti P. A study of ordered structure in acid-modified tapioca starch by ${ }^{13} \mathrm{C}$ CP/MAS solid-state NMR. Carbohydr Polym, 2004, 58: 383-389

[5] Sevenou O, Hill S E, Farhat I A, Mitchell J R. Organisation of the external region of the starch granule as determined by infrared spectroscopy. Int J Biol Macromol, 2002, 31: 79-85

[6] Chung H Y, Hoover R, Liu Q. The impact of single and dual hydrothermal modifications on the molecular structure and physicochemical properties of normal corn starch. Int J Biol Macromol, 2009, 44: 203-210

[7] Rubens P, Snauwaert J, Heremans K, Stute R. In situ observation of pressure-induced gelation of starches studied with FTIR in the diamond anvil cell. Carbohydr Polym, 1999, 39: 231-235

[8] Li Y(李玥), Zhong F(钟芳), Ma J-G(麻建国), Gu X-H(顾小红). Spectra analysis on rice starches from different varieties during pasting. Acta Polymer Sin (高分子学报), 2008, (7): 720-725 (in Chinese with English abstract)

[9] Li J H, Vasanthan T, Hoover R, Rossnagel B G. Starch from hull-less barley: V. In-vitro susceptibility of waxy, normal, and high-amylose starches towards hydrolysis by alpha-amylases and amyloglucosidase. Food Chem, 2004, 84: 621-632

[10] Wei C X, Xu B, Qin F L, Yu H G, Chen C, Meng X L, Zhu L J, Wang Y P, Gu M H, Liu Q Q. C-type starch from high-amylose rice resistant starch granules modified by antisense RNA inhibi- tion of starch branching enzyme. J Agric Food Chem, 2010, 58: 7383-7388

[11] Konik-Rose C, Thistleton J, Chanvrier H, Tan I, Halley P, Gidley M, Kosar-Hashemi B, Wang H, Larroque O, Ikea J, McMaugh S, Regina A, Rahman S, Morell M, Li Z. Effects of starch synthase IIa gene dosage on grain, protein and starch in endosperm of wheat. Theor Appl Genet, 2007, 115: 1053-1065

[12] Shingel K I. Determination of structural peculiarities of dexran, pullulan and $\gamma$-irradiated pullulan by Fourier-transform IR spectroscopy. Carbohydr Res, 2002, 337: 1445-1451

[13] Smits A L M, Ruhnau F C, Wliegenthart J F G, van Soest J J G. Ageing of starch based systems as observed with FT-IR and solid state NMR spectroscopy. Starch, 1998, 50: 478-483

[14] Wei C X, Qin F L, Zhou W D, Yu H G, Xu B, Chen C, Zhu L J, Wang Y P, Gu M H, Liu Q Q. Granule structure and distribution of allomorphs in C-type high-amylose rice starch granule modified by antisense RNA inhibition of starch branching enzyme. $J$ Agric Food Chem, 2010, 58: 11946-11954

[15] Wei C X, Qin F L, Zhou W D, Xu B, Chen C, Chen Y F, Wang Y P, Gu M H, Liu Q Q. Comparison of the crystalline properties and structural changes of starches from high-amylose transgenic rice and its its wild type during heating. Food Chem, 2011, 128: 645-652

[16] van Soest J J G, Tournois H, de Wit D, Vliegenthart J F G. Short-range structure in (partially) crystalline potato starch determined with attenuated total reflectance Fourier-transform IR spectroscopy. Carbohydr Res, 1995, 279: 201-214 